\title{
Es gibt Ausnahmen
}

\author{
GEORG VOBRUBA
}

$N$ in, ich möchte niemandem Unrecht tun. Darum werde ich betonen, dass es Ausnahmen gibt.

Die Entwicklung der Forschung und Diskussion zur Arbeitsmarkt- und Sozialpolitik ist eine Erfolgsgeschichte. Dass Arbeit und soziale Sicherheit zentrale Themen der Gesellschaftspolitik und der Sozialwissenschaften sind, musste nach dem Zweiten Weltkrieg erst mühsam wiederentdeckt werden. Die Diskurstraditionen der Zwischenkriegszeit waren verloren gegangen. Es gab Ausnahmen. In den 1970er Jahren dann nahm die Forschung einen beeindruckenden Aufschwung. Es ging um Grundsätzliches: Lebt man, um zu arbeiten, oder arbeitet man, um zu leben? Wie formieren sich grundlegende arbeitsbezogene Interessen? Wie sieht die Zukunft der Arbeit aus? Was bewirkte die Entstehung von Sozialpolitik und was treibt sie an? Welche Funktionen erfüllt Sozialpolitik im Kapitalismus? Wie stehen Arbeit und soziale Sicherheit zueinander? Wie lässt sich das Projekt Wohlfahrtsstaat weiterentwickeln?

Ein Charakteristikum jener Diskussionen war ein gewisser - und wie ich glaube: sympathischer - Größenwahn. Es wurden große Fragen gestellt, es wurde mit umfassenden Begriffen hantiert, und es dominierten pauschale Antworten. Es gab Ausnahmen, aber in der Regel ging es um die Arbeit, den Kapitalismus, den Wohlfahrtsstaat. Rückblickend scheint mir das größte Verdienst dieser Diskussionen zu sein, dass große Theoriefragen gestellt und ein großzügiger Themenrahmen für die weitere Forschung abgesteckt wurden.

Von hier aus professionalisierte sich die Arbeits- und Sozialpolitikforschung zusehends, was zu zunehmender Differenzierung der Forschungsthemen und zu zunehmender Arbeitsteilung in der Forschung führte. Nun ging es nicht mehr um die Arbeit und die Sozialpolitik, sondern um unterschiedliche Arbeitsformen und unterschiedliche Teilbereiche von Sozialpolitik. Dazu kam die Entdeckung, dass es unterschiedliche Typen von Wohlfahrtsstaaten gibt. Das mag heute selbstverständlich klingen, aber in der zweiten Hälfte der 80er Jahre war das neu. Selbstverständlich, es gab Ausnahmen. Die zunehmende Differenzierung der Forschung erhielt noch dadurch Auftrieb, dass sich ihr Gegenstand selbst immer mehr ausdifferenzierte. Es wurden eben nicht nur Phänomene berücksichtigt, über die man zuvor großzügig (großspurig) hinweggesehen hatte, es gab tatsächlich neue. Das war die Zeit, in der neue Arbeitsformen, atypische Beschäftigungsverhältnisse entstanden, Flexibilisierung zunahm, Inkongruenzen zwischen Arbeit und sozialer Sicherheit offensichtlich und Diskussionsthema wurden. Für die arbeits- und sozialpolitische Forschung gab es viel zu tun, und es entstanden zahlreiche wichtige Texte, klar in der Fragestellung, empirisch unterfüttert und mit konkreten Folgerungen.

Differenzierung, Arbeitsteilung und Konkretheit hatten aber ihren Preis. Die grundsätzlichen Fragen, die einst die Diskussion dominiert hatten, verschwanden. Das Interesse an einer gesellschaftstheoretischen Einbettung arbeits- und sozialpolitischer Probleme ließ stark nach. Der Blick für große Zusammenhänge wurde trübe. Man könnte den Eindruck gewinnen, dass aus der Unzulänglichkeit früherer Antworten auf die
Unzulässigkeit der früheren Fragen geschlossen wird. Aber dieser Eindruck trügt; einfach deshalb, weil den meisten die früheren Antworten und die gesamte Forschung nicht mehr bekannt sind. Es gibt Ausnahmen. Überwiegend aber findet man heute Forschung, die sich methodisch elaboriert Detailproblemen widmet: Exklusionsprozesse bei 17- bis 25-Jährigen, Prekarisierung von Alleinerziehenden, Verweildauer im Sozialtransferbezug von speziellen Gruppen, Probleme der Interaktion in Job-Centern. All diese Themen sind wichtig, und zwar aus mindestens zwei Gründen: Sie behandeln Probleme, die für die Betroffenen von existenzieller Bedeutung sind; und man kommt nur über den Weg empirischer Erforschung konkreter arbeits- und sozialpolitischer Phänomene zu einem integrierten Verständnis von Arbeit und sozialer Sicherheit im heutigen Kapitalismus. Nur: Dieser zweite Schritt wird nicht getan, die Einbettung der Forschung in größere Zusammenhänge fehlt. Es gibt Ausnahmen.

Man kann die Gegenprobe machen. Welche Rolle spielen Arbeit und soziale Sicherheit in den gegenwärtigen Angeboten zu einer Theorie der Gesellschaft? Keine. Es gibt kaum Ausnahmen. Im Zuge ihrer Professionalisierung ist die wechselseitige Anschlussfähigkeit von Arbeits- und Sozialpolitikforschung und Gesellschaftstheorie verloren gegangen. Das ist für beide Seiten schlecht. Die Arbeits- und Sozialpolitikforschung bezahlt damit, dass sie meist über recht einfache Ziel-Mittel-Relationen, gerne normativ aufgehübscht, nicht hinauskommt. Das Verständnis von Arbeitsteilung zwischen Wissenschaft und Politik, das dahinter steht, kann man so stilisieren: Wir zeigen, wo die Probleme sind, ihr tut etwas dagegen. Darüber hätte man vor ein paar Jahrzehnten nur den Kopf geschüttelt. Und die Ansätze zu Gesellschaftstheorien verkennen die Zentralität von Arbeit und sozialer Sicherheit im Gesellschaftsverständnis der Leute, sind also ungeschützt der Gefahr ausgesetzt, an der Realität der Menschen weit vorbeizugehen. Es gibt Ausnahmen.

Was tun? Der erreichte Stand der Professionalisierung, Differenzierung und Spezialisierung der Forschung lässt sich nicht zurücknehmen. Wenn das so ist und wenn man zugleich auf eine theoriegeleitete Gesamtsicht nicht verzichten will, dann muss man die Zusammenfassung der arbeitsteiligen Forschung auch im Rahmen der Arbeitsteilung denken und organisieren. Es muss Spezialisten für allgemeine, umfassende Fragestellungen geben. Weder die Forschungsinstitute noch die Forschungsförderung noch die wenigen verbliebenen Sozialpolitiklehrstühle berücksichtigen dies. Es gibt Ausnahmen. Aber zu wenige.

GEORG VOBRUBA, Prof. Dr., lehrt am Institut für Soziologie der Universität Leipzig und ist Vorstandsmitglied der Deutschen Gesellschaft für Soziologie.

vobruba@uni-leipzig.de 